\title{
INFRARED SPECTROSCOPY OF COMETARY PARENT MOLECULES
}

\author{
H. A. WEAVER \\ Space Telescope Science Institute \\ g700 San Martin Drive \\ Baltimore, MD 21218, USA \\ H. P. LARSON \\ Department of Planetary Sciences \\ University of Arizona \\ Tucson, AZ 85721, USA
}

\author{
M. J. MUMMA \\ Planetary Systems Branch \\ NASA Goddard Space Flight Center \\ Greenbelt, MD 20771, USA
}

\begin{abstract}
Most cometary parent molecules do not strongly fluoresce at ultraviolet and visible wavelengths, and some do not possess permanent electric dipole moments, preventing their study in the radio region as well. However, many of these molecules have strong ro-vibrational transitions in the near infrared $(\lambda \sim 2-5 \mu \mathrm{m})$. Since the solar flux at these wavelengths is quite strong, parent molecules in cometary comae can be probed directly via fluorescence in these infrared transitions. The feasibility of this approach was convincingly demonstrated by the detection of $\mathrm{H}_{2} \mathrm{O}$ in comet Halley (1986 III) from the Kuiper Airborne Observatory and by the detection of $\mathrm{H}_{2} \mathrm{O}, \mathrm{CO}_{2}$, and $\mathrm{H}_{2} \mathrm{CO}$ using an infrared spectrometer $(I K S)$ on VEGA. Tentative detections of near infrared lines of $\mathrm{CH}_{4}$ were also reported during ground-based and airborne observations of comets Halley and Wilson (1987 VII). High resolution spectroscopy of the infrared water transitions has yielded a wealth of new information on cometary physics: the absolute line intensities and spatial brightness profiles are used to determine water production rates and lifetimes, the relative line intensities probe the kinetic temperature profile in the coma, the line widths and line positions shed light on coma outflow dynamics, and the temporal variability in the lines provides information on the structure of the nucleus. These observations also allow the determination of the water ortho-to-para ratio, which may provide fundamental insight into the origin and/or evolutionary history of cometary nuclei. Similar observations of other molecules (those mentioned above plus others) will provide important complementary data and will also allow us to compile a volatile inventory for cometary nuclei, but such observations are extremely difficult due to the low abundances of these molecules ( $\leq 10 \%$ relative to water) and the limitations of present infrared facilities. Recent advances in infrared instrumentation promise to extend sensitivities for parent molecule searches to relative abundances well below $1 \%$, especially if cooled, Earth-orbiting facilities are available.
\end{abstract}




\section{Introduction}

The near infrared region of the spectrum (defined here as $\lambda \sim 2-5 \mu \mathrm{m}$ ) is fertile ground for studying cometary parent molecules. Except for homonuclear molecules, nearly all of the molecules that have been proposed as constituents of cometary nuclei have strong vibrational transitions at these wavelengths. Figure 1 shows the positions of some vibrational fundamental bands of various parent molecules. Also shown is the continuum solar flux (Labs and Neckel 1968) and infrared photometry (which primarily measures grain radiation) for a bright comet (Ney 1974). The latter two curves graphically illustrate why the region between 2 and $5 \mu \mathrm{m}$ is particularly well-suited for probing parent molecules: there is abundant solar flux for exciting infrared fluorescence while the contaminating influence of grain radiation is minimized. Thus, fluorescence in molecular lines from parent molecules can easily stand out above the grain radiation when observing comets with high spectral resolution instruments.

The emission rates, or "g-factors", for the fundamental bands of some parent molecules are given in Table 1. (Although not a parent molecule, $\mathrm{OH}$ is included as well due to its continuing importance in cometary investigations.) While these values are considerably smaller than typical g-factors in the visible, they are comparable to some of the stronger ultraviolet $\mathrm{g}$-factors. More importantly, these g-factors are large enough to produce observable emissions from comets under favorable circumstances.

TABLE 1. Infrared fluorescence rates at $1 \mathrm{AU}$

\begin{tabular}{|c|c|c|c|c|}
\hline Molecule & Transition & $\begin{array}{l}\text { Band } \\
\left(\mathrm{cm}^{-1}\right)\end{array}$ & $\begin{array}{l}\text { rigin } \\
(\mu \mathrm{m})\end{array}$ & $\begin{array}{c}\text { Band g-factor } \\
\left(10^{-4} \text { photons }\right. \\
\left.\mathrm{s}^{-1} \text { molecule }{ }^{-1}\right)\end{array}$ \\
\hline $\mathrm{H}_{2} \mathrm{O}$ & $\nu_{3}$ & 3756 & 2.66 & 2.8 \\
\hline${ }^{*} \mathrm{OH}$ & $(1,0)$ & 3570 & 2.80 & 1.1 \\
\hline $\mathrm{NH}_{3}$ & $\nu_{1}$ & 3337 & 3.00 & 0.3 \\
\hline $\mathrm{HCN}$ & $\nu_{3}$ & 3311 & 3.02 & 3.4 \\
\hline $\mathrm{CH}_{4}$ & $\nu_{3}$ & 3019 & 3.31 & 3.4 \\
\hline $\mathrm{H}_{2} \mathrm{CO}$ & $\nu_{5}$ & 2843 & 3.52 & 3.8 \\
\hline $\mathrm{H}_{2} \mathrm{CO}$ & $\nu_{1}$ & 2783 & 3.59 & 2.9 \\
\hline $\mathrm{CO}_{2}$ & $\nu_{3}$ & 2349 & 4.26 & 27. \\
\hline $\mathrm{CO}$ & $(1,0)$ & 2143 & 4.67 & 2.4 \\
\hline OCS & $\nu_{1}$ & 2062 & 4.85 & 30. \\
\hline
\end{tabular}

*The fluorescence rate for $\mathrm{OH}$ varies with the comet's heliocentric radial velocity. The value listed above is the maximum. Direct production of $O H$ in excited vibrational states via $\mathrm{H}_{2} \mathrm{O}$ photodissociation (Crovisier 1989) is another source of $\mathrm{OH}$ emission that has not been included here. 
IR COMETARY FLUX AND SOLAR FLUX

WAVELENGTH $(\mu \mathrm{m})$

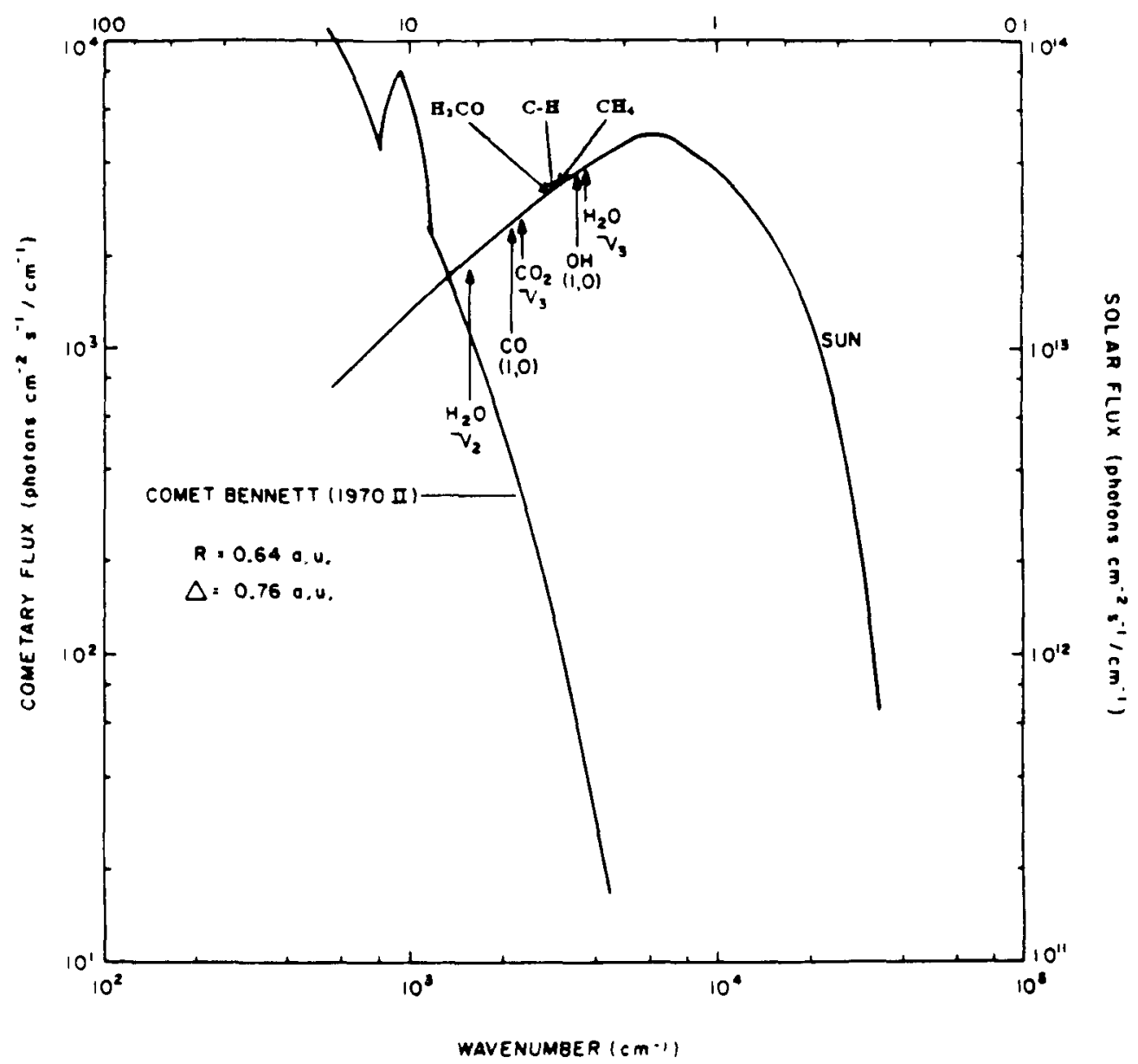

Figure 1. Strong vibrational bands of many molecules lie between 2 and $5 \mu \mathrm{m}$. The positions of such bands for several proposed cometary parent molecules are indicated. Also plotted is the solar continuum flux (Labs and Neckel 1968) at a heliocentric distance of $1 \mathrm{AU}$; this flux provides a powerful pump for infrared fluorescence by molecules in cometary comae. Detection of this fluorescence in the 2 to $5 \mu \mathrm{m}$ region is enhanced due to the decreasing flux from cometary grain radiation at these wavelengths (the broad band continuum flux from comet Bennett [Ney 1974] is plotted to illustrate this effect). The position marked " $\mathrm{C}$ $\mathrm{H}$ " represents the approximate location of $\mathrm{C}-\mathrm{H}$ stretch vibrations associated with complex hydrocarbons that may be present in comets. Although $\mathrm{OH}$ is not a parent molecule, the position of its fundamental vibration band is shown as well because of the continuing importance of $\mathrm{OH}$ in cometary investigations. This figure is adapted from Weaver and Mumma (1984). 
One of the major difficulties in cultivating the substantial potential of near infrared cometary spectroscopy is the problem of atmospheric absorption. Since most of the proposed cometary parent molecules are also constituents of the Earth's atmosphere, cometary infrared fluorescent emissions are often completely absorbed before reaching the ground. However, the use of air- and balloon-borne observatories, and of observatories in space, can overcome this obstacle. Furthermore, recent advances in infrared detector array technology promise to make infrared observations of comets an area of increasing importance. The purpose of this paper is to review the current status of near infrared spectroscopy of parent molecules and to discuss the scientific objectives of such observations.

A review of infrared cometary spectroscopy, including a discussion of grain radiation as well as gaseous emissions, has been given recently by Crovisier (1990). The reader is referred to that work for some of the details that cannot be covered in the shorter review presented here.

\section{Infrared Molecular Emissions}

The advent of infrared spectroscopy of cometary parent molecules occurred during the recent apparition of comet Halley (1986 III). The two successful near infrared spectroscopic investigations performed prior to Halley (Oishi et al. 1978, Johnson et al. 1983) did not detect any emissions from parent molecules. (New emissions were observed but these were from well-known dissociation products.) Several theoretical works (Mumma 1982, Yamamoto 1982, Encrenaz et al. 1982, Crovisier and Encrenaz 1983, Weaver and Mumma 1984, Crovisier 1984) on infrared fluorescence from cometary parent molecules were published in the early 1980's that paved the way for the first successful observations on comet Halley. In December, 1985 the $\nu_{3}$ band of the water molecule was unambiguously detected from the Kuiper Airborne Observatory (KAO) (Mumma et al. 1986), which flies above $99 \%$ of the terrestrial water vapor. In March, 1986 an infrared spectrometer on the VEGA spacecraft (the IKS experiment) observed emissions from $\mathrm{H}_{2} \mathrm{O}, \mathrm{CO}_{2}, \mathrm{H}_{2} \mathrm{CO}$, and possibly CO and OCS (Moroz et al. 1987, Combes et al. 1988). Figure 2 shows the IKS spectrum near the time of closest approach to the nucleus. In this section we discuss fluorescent emissions from parent molecules on a case-by-case basis. Special emphasis is given to the $\mathrm{H}_{2} \mathrm{O}$ results due to the extensive measurements made of this molecule.

\section{$2.1 \mathrm{H}_{2} \mathrm{O}$}

During the past two decades observers built a strong circumstantial case for $\mathrm{H}_{2} \mathrm{O}$ ice as the dominant volatile constituent in cometary nuclei. However, the apparition of comet Halley provided the first opportunity to observe the $\mathrm{H}_{2} \mathrm{O}$ molecule directly. Pre-perihelion $K A O$ observations of Halley (Mumma et al. 1986) revealed intense, solar-pumped fluorescent emission in the $\nu_{3}$ band of $\mathrm{H}_{2} \mathrm{O}$ near $\lambda \sim 2.65 \mu \mathrm{m}$. This band was subsequently observed post-perihelion from the KAO (Weaver et al. 1986) and VEGA (Moroz et al. 1987, Combes et al. 1988). Similar KAO observations were successfully conducted on comet Wilson (1987 VII) (Larson et al. 1988). Figure 3 shows examples of high resolution spectra $\left(\nu / \Delta \nu \sim 10^{5}\right)$ taken from the $K A O$ in which individual ro-vibration lines of $\mathrm{H}_{2} \mathrm{O}$ are clearly resolved. These data allow one to address a diverse range of cometary problems. 


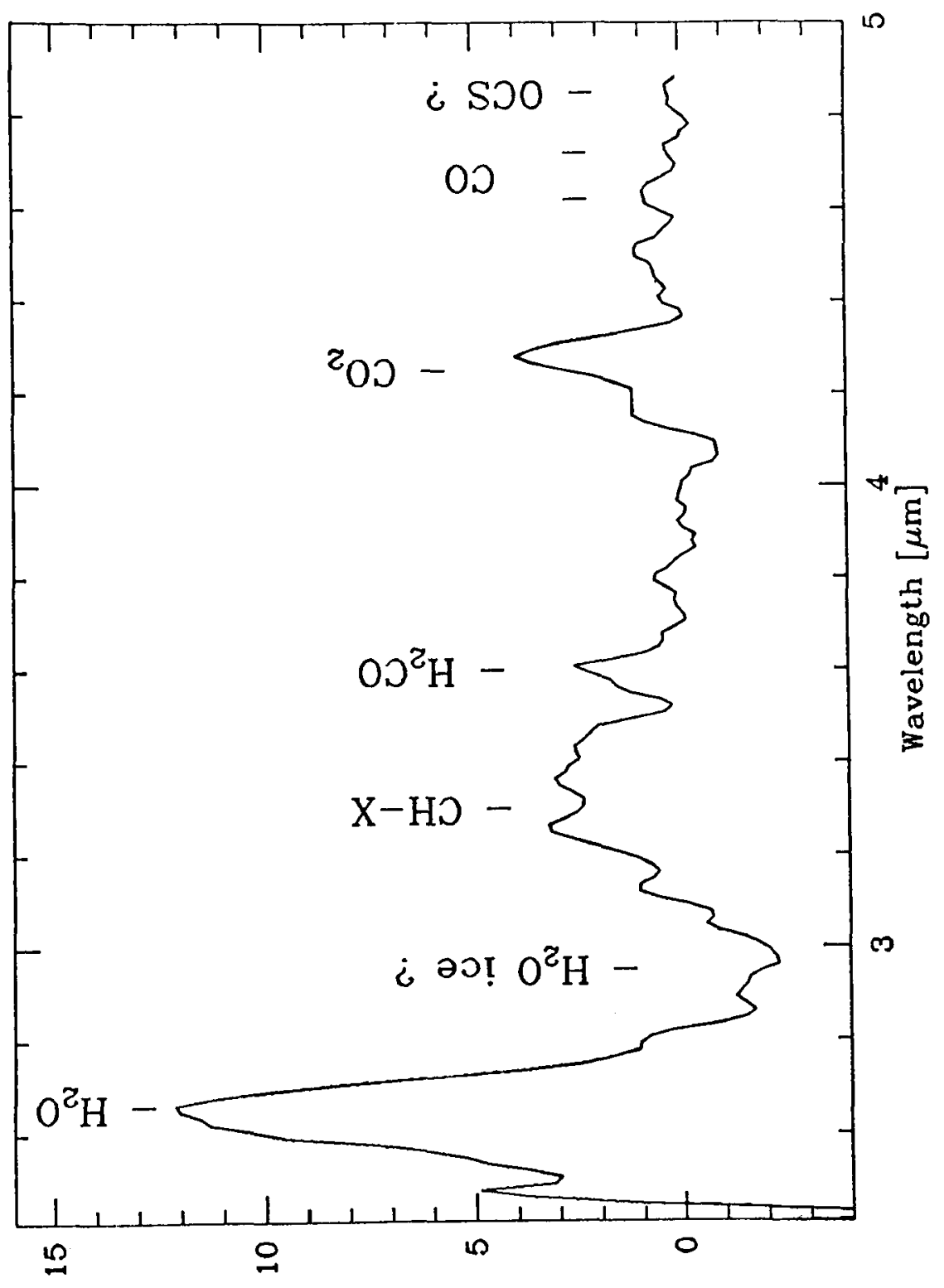

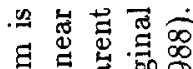

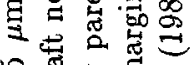

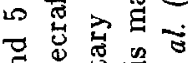

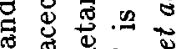

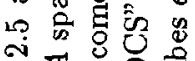

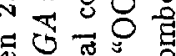
षن 跣志

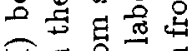
岳总 $\circ \widehat{\sigma}$ 品造禺 $己 こ$.

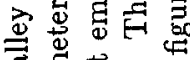

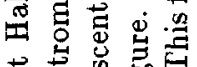
苛壱 苍造岁 ن 吾 ○ 岕䟢

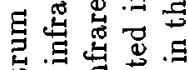

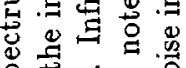
古

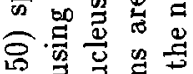
ᄀ古总出

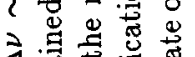

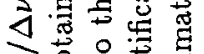

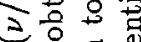

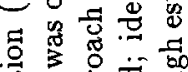
寻䓃运 율 领

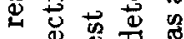

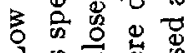
今.

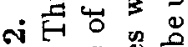

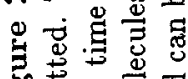

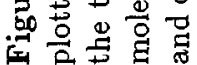

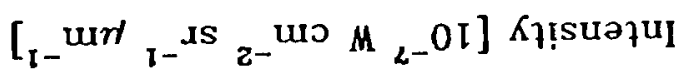



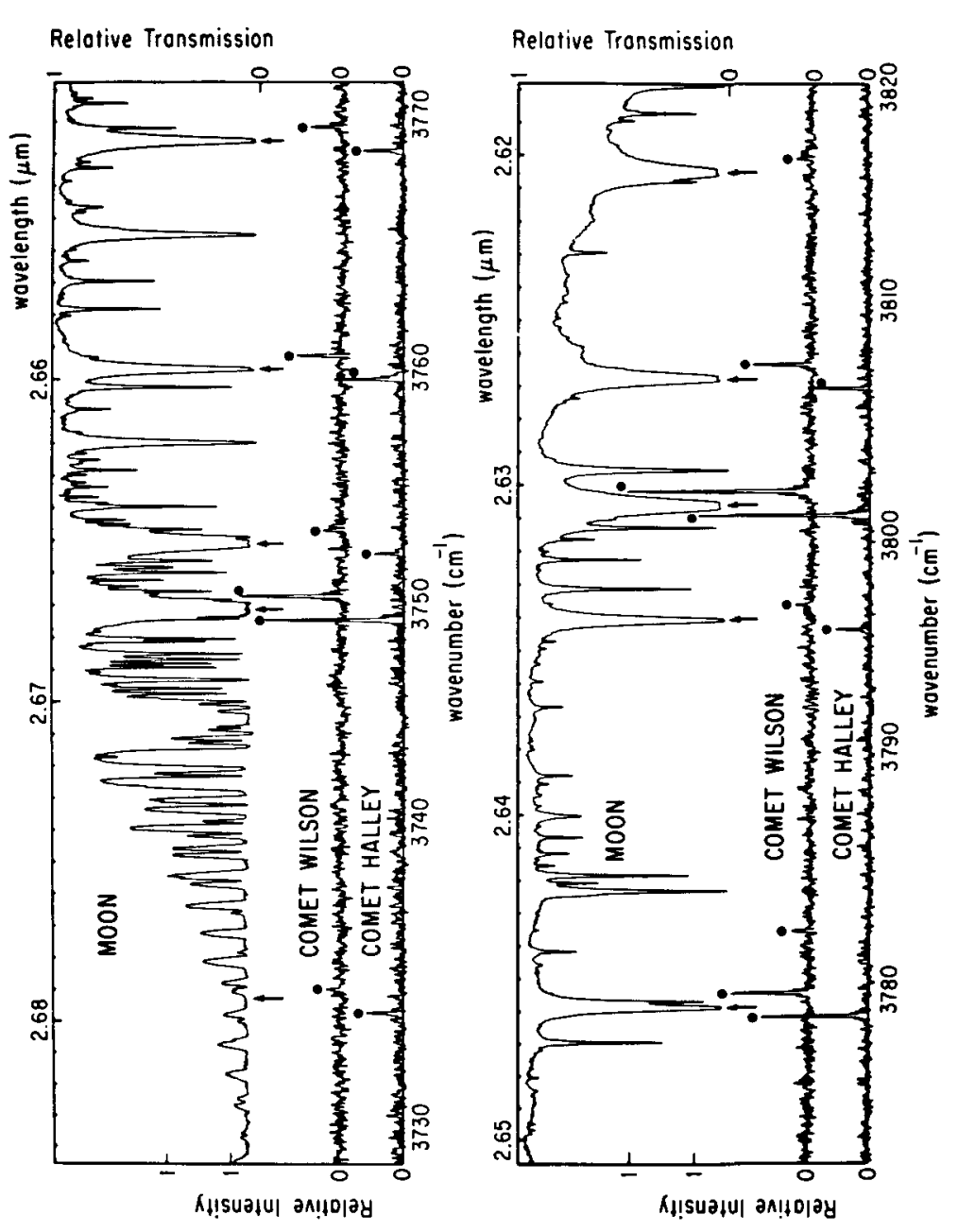

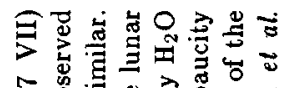

务骂些

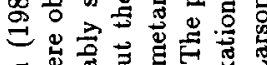

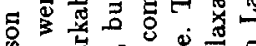

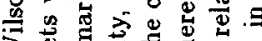

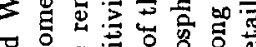

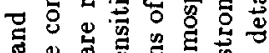

至点

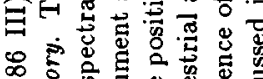

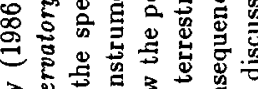

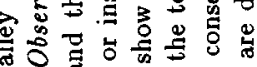

两

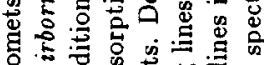

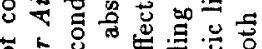

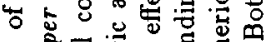

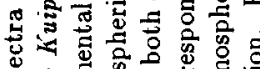

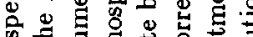

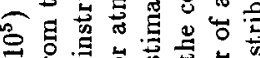

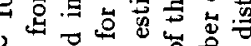

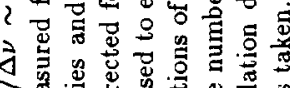

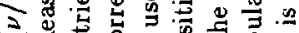

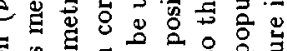

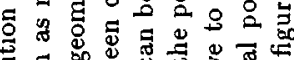

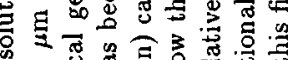

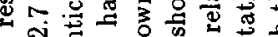

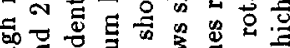

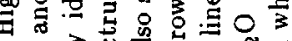

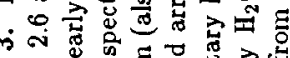

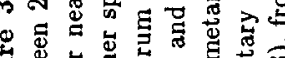

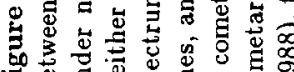

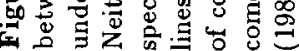


2.1.1 Coma Excitation Conditions. The kinetic temperature profile of the inner coma is determined by the competition among expansion cooling, photolytic heating, and radiative cooling by $\mathrm{H}_{2} \mathrm{O}$ molecules ( $c f$. Bockelée-Morvan and Crovisier 1987a). Collisions will cause the $\mathrm{H}_{2} \mathrm{O}$ rotational temperature to equal the gas kinetic temperature in this region of the coma. The $\mathrm{H}_{2} \mathrm{O}$ excitation in the outer coma is determined solely by radiative processes (i.e., the molecules approach fluorescence equilibrium). Since the rotational Einstein A coefficients in $\mathrm{H}_{2} \mathrm{O}$ are so large, the rotational temperature of $\mathrm{H}_{2} \mathrm{O}$ molecules in the outer coma will be exceptionally low with only the lowest energy ortho and para levels having significant populations. (This explains why cometary observations of the water maser line at $1.35 \mathrm{~cm}$ have been unsuccessful; the upper state for this transition is $\sim 640 \mathrm{~K}$ above the ground state.) The $K A O$ observations of the relative intensities of individual ro-vibrational lines of $\mathrm{H}_{2} \mathrm{O}$ yield direct information on all these processes. Sophisticated modeling of these emissions, including optically thick radiative transfer in addition to the effects mentioned above, has been developed over the past several years (Bockelée-Morvan 1987). Comparisons between theory and observation (Bockeleee-Morvan and Crovisier 1987b) indicate that expansion and radiative cooling overcome the photolytic heating, thus producing low gas kinetic temperatures $\left(T \leq 100 \mathrm{~K}\right.$ ) for distances $\geq 10^{3} \mathrm{~km}$ from the nucleus. (The observations made to date are not very sensitive to conditions within $\sim 10^{3} \mathrm{~km}$ of the nucleus.)

2.1.2 Water Production Rates and Temporal Variability. Water production rates $\left(\mathrm{Q}_{\mathrm{H}_{2} \mathrm{O}}\right)$ are used for testing sublimation models, for modeling the excitation conditions in the coma, for monitoring temporal activity, and for determining relative abundances of other species. ( $\mathrm{H}_{2} \mathrm{O}$ is the "standard" to which trace species are compared.) Until the Halley apparition, observations of $\mathrm{H}_{2} \mathrm{O}$ dissociation products (e.g., $\mathrm{OH}, \mathrm{O}$, and $\mathrm{H}$ ) were used to derive $\mathrm{Q}_{\mathrm{H}_{2} \mathrm{O}}$. In principle, direct observations of $\mathrm{Q}_{\mathrm{H}_{2} \mathrm{O}}$, like those made from the $K A O$, should yield more reliable determinations.

The $K A O$ Halley $\mathrm{H}_{2} \mathrm{O}$ observations showed a large pre- to post-perihelion asymmetry in $\mathrm{Q}_{2} \mathrm{O}$ demonstrating that Halley was about a factor of 5 to 7 more active post-perihelion at $\mathrm{R}=1 \mathrm{AU}$ than at the same heliocentric distance pre-perihelion (Weaver et al. 1987). Short-term temporal activity was also observed in Halley on timescales ranging from a few days to two hours (Mumma et al. 1986, Weaver et al. 1986), and possibly even less (Larson et al. 1990).

The temporal variability in Halley reveals indirect information on the structure of the nucleus. The rather smooth, quasi-periodic behavior in Halley's visible and ultraviolet brightness (Millis and Schleicher 1986, McFadden et al. 1987) has been attributed to the rotation of discrete active areas on the nucleus surface into sunlight. The KAO infrared observations showed evidence of large, random outbursts in $Q_{H_{2} O}$ that may imply the release of a significant amount of internal energy from the nucleus, as might occur, for example, during the transformation of nuclear ice from amorphous to crystalline form (Larson et al. 1990).

The water production rates derived from the $K A O$ Halley observations were quite different (up to factor of 3) from those derived from International Ultraviolet Explorer (IUE) observations of $\mathrm{OH}$ (Weaver et al. 1987; radio OH observations are not discussed here since they are compared with the IUE OH observations by Gérard elsewhere in this volume), but at least some of this difference may be attributed to the large absolute calibration error 
$( \pm 50 \%)$ associated with the $K A O$ observations. For comet Wilson, the $K A O$ errors were smaller $( \pm 30 \%)$ and the water production rates derived from the $K A O$ and $I U E$ observations are in excellent agreement (Larson et al. 1988, Roettger et al. 1989). Neither the KAO nor the $I U E$ observations detected any significant temporal activity in comet Wilson; it has been suggested that the large temporal variability in Halley could produce an "apparent" discrepancy when comparing $\mathrm{Q}_{\mathrm{H}_{2} \mathrm{O}}$ derived from $\mathrm{H}_{2} \mathrm{O}$ and $\mathrm{OH}$ observations (Weaver et al. 1986).

2.1.3 Spatial Brightness Profile and Water Lifetime. Comet Halley was bright enough that some limited mapping of the $\mathrm{H}_{2} \mathrm{O}$ spatial brightness distribution was attempted during the $K A O$ observations. A rather large sunward-tailward brightness asymmetry was observed, demonstrating clearly that $\mathrm{H}_{2} \mathrm{O}$ was being released primarily into the sunward-facing hemisphere (Weaver et al. 1986).

The $\mathrm{H}_{2} \mathrm{O}$ spatial brightness profile can be used to derive a water lifetime. Initially a surprisingly low value was retrieved which was about three times shorter than the calculated photochemical lifetime (Weaver et al. 1986). However, recent work has shown that the use of a constant $\mathrm{H}_{2} \mathrm{O}$ outflow velocity in the radial outflow model used to interpret the observations (as was done in the original analysis) will significantly underestimate the $\mathrm{H}_{2} \mathrm{O}$ lifetime and that the $K A O$ data can be fit with the "canonical" lifetime (Hu and Larson 1990). We point out that the large temporal variability that took place during the mapping observations adds a further complication to any analysis of these spatial brightness profiles.

2.1.4 Kinematics. Line shape data from the high resolution $K A O$ observations provide a "spectroscopic picture" of the $\mathrm{H}_{2} \mathrm{O}$ outflow. The absolute line positions are diagnostic of asymmetries in the outflow, while the line widths yield direct information on the outflow velocity. Results from comet Halley indicate that the outflow velocity pre-perihelion was significantly less than the post-perihelion velocity $\left(0.6-1 \mathrm{~km} \mathrm{~s}^{-1}\right.$ vs. $\left.1.4-2 \mathrm{~km} \mathrm{~s}^{-1}\right)$ even though both observations were taken at approximately the same heliocentric distance (Larson et al. 1986, Larson et al. 1987). Both pre- and post-perihelion data show evidence for strong asymmetry in the outflow with the post-perihelion velocity field being particularly complex. The KAO observations of comet Wilson also revealed asymmetric outflow and a rather large outflow velocity $\left(\sim 1.5 \mathrm{~km} \mathrm{~s}^{-1}\right.$; Larson et al. 1988).

2.1.5 Ortho-to-Para Ratio. Since transitions between the ortho and para "species" of $\mathrm{H}_{2} \mathrm{O}$ are forbidden for both radiative and collisional processes, the ortho-to-para ratio (OPR) cannot be modified in the coma. Thus, the OPR presumably yields direct information on the history of the nucleus (Mumma et al. 1987, Mumma et al. 1988). For example, a "new" comet whose outer layers have been modified by cosmic ray bombardment during its storage in the Oort Cloud might be expected to have an $\mathrm{OPR}=3$, the ratio of the statistical weights of the two species. Periodic comets that have lost their outer layers may have a different OPR. If the equilibration time for the OPR is very long (i.e., as long as the age of the solar system), then the OPR in periodic comets may reflect their formation temperatures and will be significantly less than 3 if this temperature is $\leq 40 \mathrm{~K}$. If $\mathrm{OPR}$ equilibration in $\mathrm{H}_{2} \mathrm{O}$ ice is relatively fast, then the OPR may simply track the equilibrium temperature of the nucleus in its orbit, in which case the OPR would be 3 for most comets observed near perihelion. 
Recent KAO observations of comets Halley and Wilson have yielded rather intriguing results on the OPR. The value derived for the "new" comet Wilson is significantly larger than that derived for the periodic comet Halley (Mumma et al. 1988). While this latter statement appears secure, it must be emphasized that the derived numerical values for the OPR are model dependent and may require revision as models are improved. Although individual ortho and para $\mathrm{H}_{2} \mathrm{O}$ lines are cleanly separated in the $K A O$ spectra, some important lines in the $\mathrm{H}_{2} \mathrm{O}$ band are obscured by atmospheric absorption and this also affects the retrieval of the OPR. Moreover, reliable interpretation of the OPR in comets must await further laboratory data on the interconversion of the ortho and para species of $\mathrm{H}_{2} \mathrm{O}$ in the ice phase.

\subsection{LINEAR MOLECULES: $\mathrm{CO}, \mathrm{CO}_{2}, \mathrm{HCN}$, and OCS}

Crovisier (1987) has discussed procedures for calculating g-factors for linear molecules, including all the species given in the section title. The reader is referred to that paper for details. Below we focus on the status of observational searches for these species.

Prior to Halley, neutral CO had been observed directly in only two comets: the CO fourth positive group near $\lambda \sim 1500 \AA$ was detected during sounding rocket observations of comet West (1976 VI) (Feldman and Brune 1977) and during IUE observations of comet Bradfield (1979 X) (A'Hearn and Feldman 1982). The CO $(1,0)$ fundamental band near 4.7 $\mu \mathrm{m}$ is observable from the ground if the geocentric radial velocity of the comet is reasonably large $\left(\geq 15 \mathrm{~km} \mathrm{~s}^{-1}\right)$. Although a sensitive search was made on comet IRAS-Araki-Alcock (1983 VII) (Chin and Weaver 1984), the CO infrared band was not detected. The IKS spectrum shows evidence for $\mathrm{CO}$ (Combes et al. 1988; see Figure 2) but the signal-to-noise is poor. The relative abundance limit from the $I K S \mathrm{CO}$ observation is $\mathrm{CO} / \mathrm{H}_{2} \mathrm{O} \sim 5 \%$, but the IKS data measure only the component of $\mathrm{CO}$ sublimating directly from the nucleus. The Giotto NMS experiment showed that the total CO production rate is $\sim 15 \%$ of the $\mathrm{H}_{2} \mathrm{O}$ production rate, with most of the $\mathrm{CO}$ in the coma being derived from an extended source (e.g., from evaporation of organic grains or from photodestruction of $\mathrm{H}_{2} \mathrm{CO}$ ). Thermal background limits the sensitivity of $\mathrm{CO}$ searches and definite detections of its infrared band might have to await the development of a cooled, orbiting infrared observatory, or of high resolution, cryogenic spectrometers on Earth-based or airborne observatories.

Neutral $\mathrm{CO}_{2}$ was observed for the first time in a comet by the $I K S$, which detected the $\mathrm{CO}_{2} \nu_{3}$ fundamental band near $4.3 \mu \mathrm{m}$ in comet Halley (Moroz et al. 1987, Combes et al. 1988; see Figure 2). Although $\mathrm{CO}_{2}$ is a trace constituent of the nucleus ( $\sim 4 \%$ relative to $\mathrm{H}_{2} \mathrm{O}$ in Halley), its $\nu_{3}$ band is exceptionally strong, making $\mathrm{CO}_{2}$ a promising target for future study. However, $\mathrm{CO}_{2}$ in the terrestrial atmosphere produces very strong absorption even up to balloon altitudes. Thus, systematic observations of $\mathrm{CO}_{2}$ may also have to await the development of the appropriate infrared satellite observatory.

The $\mathrm{J}=1-0$ rotational transition of the $\mathrm{HCN}$ molecule $(\lambda \sim 3.4 \mathrm{~mm})$ was observed extensively in Halley (Despois et al. 1986, Schloerb et al. 1987), but its infrared fundamental band near $3 \mu \mathrm{m}$ has never been observed. The g-factor of this band is fairly large (see Table $1)$, but the $\mathrm{HCN}$ abundance in comets $\left(\sim 0.1 \%\right.$ relative to $\left.\mathrm{H}_{2} \mathrm{O}\right)$ rules out its detection until significant improvements are achieved in instrumental sensitivities.

The $\nu_{1}$ band of OCS near $4.9 \mu \mathrm{m}$ is also exceptionally strong. There is marginal evidence 
for the presence of OCS in the IKS spectrum (Combes et al. 1988; see Figure 2), but this result is more properly interpreted as an upper limit. From the infrared observation and from ultraviolet observations of atomic sulfur and other sulfur-bearing compounds ( $c f$. Feldman, this volume), a conservative upper limit to the OCS abundance in comets is $\sim 1 \%$. Thus, future infrared observations of this molecule will be extremely difficult.

\section{$2.3 \mathrm{H}_{2} \mathrm{CO}$}

Formaldehyde is frequently observed in molecular clouds via its radio transitions, but it had never been observed in a comet prior to Halley. There is a significant emission feature in the $I K S$ spectrum that coincides with the position of the $\nu_{5}$ and $\nu_{1}$ bands of $\mathrm{H}_{2} \mathrm{CO}$ (Combes et al. 1988; see Figure 2). However, the IKS team claimed that part of the emission was spurious and fit the remainder with a model $\mathrm{H}_{2} \mathrm{CO}$ spectrum having a rotational temperature of $300 \mathrm{~K}$. A reanalysis of these data (Mumma and Reuter 1989) indicates that the entire emission, including the "spurious" peak, is well-fit by an $\mathrm{H}_{2} \mathrm{CO}$ spectrum having a much colder rotational distribution. The $\mathrm{H}_{2} \mathrm{CO}$ abundance derived from both analyses agree and give a surprisingly high value $\left(\sim 4 \%\right.$ relative to $\left.\mathrm{H}_{2} \mathrm{O}\right)$. A rotational line of $\mathrm{H}_{2} \mathrm{CO}$ (the $1_{11}-1_{10}$ transition at $\lambda \sim 6 \mathrm{~cm}$ ) was detected from the Very Large Array (VLA) radio telescope from which a relative $\mathrm{H}_{2} \mathrm{CO}$ abundance of $\sim 1.5 \%$ was derived (Snyder et al. 1989). Moreover, modeling of this radio emission indicates that at least some of the observed $\mathrm{H}_{2} \mathrm{CO}$ was derived from an extended source (e.g., polyoxymethylene). Recent analysis of Giotto NMS data also indicates that the source of $\mathrm{H}_{2} \mathrm{CO}$ in the coma of Halley is extended spatially (Krankowsky, this volume), very much like the case of CO.

The infrared $\mathrm{H}_{2} \mathrm{CO}$ bands lie in a spectral region that is accessible from the ground, but no definite detections have been reported, although several sensitive searches have been made (Baas et al. 1986, Danks et al. 1987, Brooke et al. 1989). In fact, strong evidence indicates that the emissions observed near $3.5 \mu \mathrm{m}$ and $3.6 \mu \mathrm{m}$ (where the $\nu_{5}$ and $\nu_{1}$ bands occur) cannot be explained by $\mathrm{H}_{2} \mathrm{CO}$ (Brooke et al. 1989). These negative results may indicate either that the $\mathrm{H}_{2} \mathrm{CO}$ abundance is temporally variable in a comet, and/or that the abundance varies from comet to comet. A broad cometary emission centered near 3.4 $\mu \mathrm{m}$ and identified as $\mathrm{C}-\mathrm{H}$ stretch vibrations in organic grains contaminates the region where the $\mathrm{H}_{2} \mathrm{CO}$ bands occur, further complicating the detection of $\mathrm{H}_{2} \mathrm{CO}$. Future ground-based searches should yield valuable insight into these questions.

\section{$2.4 \mathrm{CH}_{4}$ and $\mathrm{NH}_{3}$}

Spectroscopic observations of the $\nu_{3}$ band of $\mathrm{CH}_{4}$ near $\lambda \sim 3.3 \mu \mathrm{m}$ provide a direct probe of methane in comets. The $\mathrm{R}(1)$ line of this band was marginally detected in comet Halley (Kawara et al. 1988), while an average over six R-branch lines in this band gave a tentative detection during KAO observations of comet Wilson (Larson et al. 1988). KAO observations of comet Halley yielded only a sensitive upper limit to the $\mathrm{CH}_{4}$ abundance (Drapatz et al. 1987). High spectral resolution $(\nu / \Delta \nu>1000)$ was used during these investigations in order to overcome problems associated with contamination from broad-band cometary emissions (e.g., thermal radiation from grains including the feature centered near $3.4 \mu \mathrm{m}$ that is thought to be associated with organic grains). 
The interpretation of these results in terms of the $\mathrm{CH}_{4}$ abundance is somewhat problematical. The derived abundance can vary significantly depending on the choice of the "effective" excitation temperature of the $\mathrm{CH}_{4}$ molecules in the coma, especially if only one line is used (e.g., the abundance derived in Kawara et al. varies by a factor of $\sim 6$ as the excitation temperature is varied from $50 \mathrm{~K}$ to $200 \mathrm{~K}$ ). We simply note that the KAO observations of $\mathrm{H}_{2} \mathrm{O}$ discussed earlier indicate that the appropriate excitation temperature of $\mathrm{CH}_{4}$ is probably $\leq 100 \mathrm{~K}$, implying that the $\mathrm{CH}_{4}$ abundance is $\leq 0.5 \%$ in comet Halley and $\leq 2 \%$ in comet Wilson. The $\mathrm{CH}_{4}$ abundance in comet Halley deduced indirectly from analysis of data from the Giotto IMS experiment is about $2 \%$, but the uncertainties in this case are very large (up to a factor of four in either direction) (Allen et al. 1987).

The question about the excitation temperature for $\mathrm{CH}_{4}$ is particularly interesting because $\mathrm{CH}_{4}$ has no allowed rotational transitions, and fluorescence in the $\nu_{3}$ vibrational lines does not efficiently redistribute the population of the ground-state rotational levels (Drapatz et al. 1987). Thus, the rotational level populations in $\mathrm{CH}_{4}$ that are established by collisions in the inner coma remain "frozen" throughout much of the outer coma, so that a measurement of the relative intensities of the vibrational lines in the $\nu_{3}$ band yields a direct measurement of the coma kinetic temperature. Unfortunately, the sensitivity required to exploit this effect is slightly beyond the capabilities of current instrumentation, except possibly for very bright comets.

There has only been one direct observation of $\mathrm{NH}_{3}$ in a comet: a radio line was marginally detected in comet IRAS-Araki-Alcock (1983 VII) (Altenhoff et al. 1983). From observations of the $\mathrm{NH}_{2}$ radical in the visible, we know that the $\mathrm{NH}_{3}$ abundance in comets is probably $<1 \%$ (Wyckoff et al. 1990). This, coupled with the fact that the g-factor for the $\mathrm{NH}_{3} \nu_{1}$ band is small, implies that $\mathrm{NH}_{3}$ will probably not be detected via its near infrared band in the near future. Although the $\nu_{2}$ band near $10 \mu \mathrm{m}$ is stronger, this is a more difficult spectral region in which to observe. Heterodyne observations of the $10 \mu \mathrm{m}$ band in comet IRAS-Araki-Alcock yielded upper limits on the $\mathrm{NH}_{3}$ abundance on the order of $\sim 10 \%$ (Mumma et al. 1984).

\section{Conclusion}

Clearly some very interesting results have already emerged during near infrared spectroscopic observations of cometary parent molecules. The work so far has concentrated primarily on the $\mathrm{H}_{2} \mathrm{O}$ molecule due to its high abundance in comets and favorable observing circumstances (e.g., the region of its $\nu_{3}$ fundamental band is not strongly affected by thermal background considerations, and the atmosphere shows some transparency to the cometary emissions as long as the observations are above the tropopause). Three successful observing programs on comets have been conducted from the $K A O$ and more are anticipated. The ability to deploy the $K A O$ on relatively short notice for new targets of opportunity is a potential important advantage of the airborne cometary program.

However, the challenge facing the IR community is to extend the cometary work to the trace molecular constituents of cometary nuclei. Only then will we be able to compile a detailed inventory of cometary volatiles that can then be used to test theories of planetary system formation and evolution. The initial exciting results from the VEGA IKS experiment have given us a glimpse of what is attainable in the future. The introduction of 
extremely sensitive infrared arrays during the next few years, and the use of cryogenic high resolution spectrometers on the $K A O$ and its successor, the Stratospheric Observatory for Infrared Astronomy (SOFIA), should bring at least several trace cometary parent molecules within detection limits. In addition, the deployment of Earth-orbiting observatories possessing near infrared capabilities, such as the Infrared Space Observatory (ISO), the Space Infrared Telescope Facility (SIRTF), and the Hubble Space Telescope (HST) (on HST the "second generation" infrared instrument is scheduled for orbit insertion about five years after launch), should further extend our grasp, especially in those cases where atmospheric absorption remains a problem even at airplane altitudes. Besides providing fundamental insight into the physics of cometary comae, near infrared cometary spectroscopy promises to improve significantly our knowledge of the trace volatile composition of cometary nuclei, allowing us to address some of the cosmogonic issues that make comets so important.

\section{Acknowledgements}

The authors thank $\mathbf{J}$. Crovisier for his comments on an earlier draft of this review. The $K A O$ observations and the analyses of the $K A O$ data were supported through NASA grants NAG-555 to the Johns Hopkins University, NAG-555 to the Space Telescope Science Institute, and NAG-555 to the University of Arizona, and through RTOP-555 to the Goddard Space Flight Center.

\section{References}

A'Hearn, M.F., and Feldman, P.D. (1982) 'Carbon in comet Bradfield (1979l)', Ap. J. Lett., 242, L187-L190.

Allen, M., et al. (1987) 'Evidence for methane and ammonia in the coma of comet P/Halley', Astron. Ap., 187, 502-512.

Altenhoff, W.J., et al. (1983) 'Radio observations of comet 1983d', Astron. Ap., 125, L19-L22.

Baas, F., Geballe, T.R., and Walther, D.M. (1986) 'Spectroscopy of the 3.4 micron emission feature in comet Halley', Ap. J. Lett., 311, L97-L101.

Bockelée-Morvan, D. (1987) 'A model for the excitation of water in comets', Astron. Ap., 181, 169-181.

Bockelée-Movan, D., and Crovisier, J. (1987a) 'The role of water in the thermal balance of the coma', in E.J. Rolfe and B. Battrick (eds.), Symposium on the diversity and similarity of comets, ESA SP-278, 235-240.

Bockelée-Morvan, D., and Crovisier, J. (1987b) 'The $2.7 \mu \mathrm{m}$ water band of comet P/Halley: interpretation of observations by an excitation model', Astron. Ap., 187, 425-430.

Brooke, T.Y., Knacke, R.F., Owen, T.C., and Tokunaga, A.T., (1989) 'Spectroscopy of emission features near $3 \mu \mathrm{m}$ in comet Wilson (1986l)', Ap. J., 336, 971-978.

Chin, G., and Weaver, H.A. (1984) 'Vibrational and rotational excitation of CO in comets: nonequilibrium calculations', Ap. J., 285, 858-869.

Combes, M., et al. (1988) 'The 2.5-12 $\mu \mathrm{m}$ spectrum of comet Halley from the IKS-VEGA experiment', Icarus, 76, 404-436. 
Crovisier, J. (1984) 'The water molecule in comets: fluorescence mechanisms and thermodynamics of the inner coma', Astron. Ap., 130, 361-372.

Crovisier, J. (1987) 'Rotational and vibrational synthetic spectra of linear parent molecules in comets', Astron. Ap. Suppl. Ser., 68, 223-258.

Crovisier, J. (1989) 'The photodissociation of water in cometary atmospheres', Astron. Ap., $213,459-464$.

Crovisier, J. (1990) 'Infrared cometary spectroscopy', in 22nd ESLAB symposium on infrared spectroscopy in astronomy, ESA SP-290, in press.

Crovisier, J., and Encrenaz, Th. (1983) 'Infrared fluorescence of molecules in comets: the general synthetic spectrum', Astron. Ap., 126, 170-182.

Danks, A.C., Encrenaz, T., Bouchet, P., Le Bertre, T., and Chalabaev, A. (1987) 'The spectrum of comet P/Halley from 3.0 to $4.0 \mu \mathrm{m}$ ', Astron. Ap., 184, 329-332.

Despois, D., et al. (1986) 'Observations of hydrogen cyanide in comet Halley', Astron. Ap., 160, L11-L12.

Drapatz, S., Larson, H.P., and Davis, D.S. (1987) 'Search for methane in comet P/Halley', Astron. Ap., 187, 497-501.

Encrenaz, Th., Crovisier, J., Combes, M., and Crifo, J.F. (1982) 'A theoretical study of comet Halley's spectrum in the infrared range', Icarus, 51, 660-664.

Feldman, P.D., and Brune, W.H. (1977) 'Carbon production in comet West 1975n', Ap. J. Lett., 209, L45-L48.

Feldman, P.D., et al. (1987) 'IUE observations of P/Halley: evolution of the ultraviolet spectrum between September 1985 and July 1986', Astron. Ap., 187, 325-328.

$\mathrm{Hu}, \mathrm{H}-\mathrm{Y}$., and Larson, H.P. (1990) 'The photochemical lifetime of $\mathrm{H}_{2} \mathrm{O}$ in cometary comae', preprint.

Johnson, J.R., Fink, U., and Larson, H.P. (1983) 'The 0.9-2.5 micron spectrum of comet West (1976 VI)', Ap. J., 270, 769-777.

Kawara, K., Gregory, B., Yamamoto, T., and Shibai, H. (1988) 'Infrared spectroscopic observation of methane in comet Halley', Astron. Ap., 207, 174-181.

Labs, D., and Neckel, H. (1968) 'The radiation of the solar photosphere from $2000 \AA$ to 100 $\mu^{\prime}$, Zs. Ap., 69, 1-73.

Larson, H.P., Mumma, M.J., Weaver, H.A., and Davis, D.S. (1986) 'Velocity-resolved observations of water in comet Halley', Ap. J. Lett., 309, L95-L99.

Larson, H.P., Mumma, M.J., and Weaver, H.A. (1987) 'Kinematic properties of the neutral gas outflow from comet P/Halley', Astron. Ap., 187, 391-397.

Larson, H.P., Weaver, H.A., Mumma, M.J., and Drapatz, S. (1988) 'Airborne infrared spectroscopy of comet Wilson (1986l) and comparisons with comet Halley', Ap. J., 338, 1106-1114.

Larson, H.P., Hu, H-Y., Mumma, M.J., and Weaver, H.A. (1990) 'Outbursts of $\mathrm{H}_{2} \mathrm{O}$ in comet $\mathrm{P} /$ Halley', Icarus, in press.

McFadden, L.A., A'Hearn, M.F., Feldman, P.D., Roettger, E.E., Edsall, D.M., and Butterworth, P.S. (1987) 'Activity of comet P/Halley on March 23-25, 1986: IUE observations', Astron. Ap., 187, 333-338.

Millis, R.L., and Schleicher, D.G. (1986) 'Rotational period of comet Halley', Nature, 324, 646-649. 
Moroz, V.I., et al. (1987) 'Detection of parent molecules in comet P/Halley from the IKS-VEGA experiment', Astron. Ap., 187, 513-518.

Mumma, M.J. (1982) 'Speculations on the infrared spectra of comets', in M.J. Mumma, K. Fox, and J. Hornstein (eds.), Vibrational-rotational spectroscopy for planetary atmospheres, NASA CP-2223, v. II, 717-744.

Mumma, M.J., Kostiuk, T., and Weaver, H.A. (1984) 'Limits on the production of $\mathrm{NH}_{3}$ in comet IRAS-Araki-Alcock', BAAS, 16,638.

Mumma, M.J., Weaver, H.A., Larson, H.P., Davis, D.S., and Williams, M. (1986) 'Detection of water vapor in Halley's comet', Science, 232, 1523-1528.

Murmma, M.J., Weaver, H.A., and Larson, H.P. (1987) 'The ortho-para ratio of water vapor in comet P/Halley', Astron. Ap., 187, 419-424.

Mumma, M., Blass, W., Weaver, H., and Larson, H. (1988) 'Measurements of the orthopara ratio and nuclear spin temperature of water in comets Halley and Wilson (1986l) and implications for their origin and evolution', in H.A. Weaver, F. Paresce and L. Danly (eds.), Poster Book from the STScI workshop on the Formation and Evolution of Planetary Systems, pp. 157-168.

Mumma, M.J., and Reuter, D. (1989) 'On the identification of formaldehyde in Halley's comet', Ap. J., in press.

Ney, E.P. (1974) 'Multiband photometry of comets Kohoutek, Bennett, Bradfield, and Encke', Icarus, 23, 551-560.

Oishi, M., et al. (1978) 'Infrared observations of comet West (1975n). I. Observational results', Publ. Astron. Soc. Japan, 30, 149-159.

Roettger, E.E., Feldman, P.D., A'Hearn, M.F., FesM.C., McFadden, L.A., and Gilmozzi, R. (1989) 'IUE observations of the evolution of comet Wilson (1986): comparison with P/Halley', Icarus, 80, 303-314.

Schloerb, F.P., Kinzel, W.M., Swade, D.A., and Irvine, W.M. (1987) 'Observations of HCN in comet P/Halley', Astron. Ap., 187, 475-480.

Snyder, L.E., Palmer, P., and De Pater, I. (1989) 'Radio detection of formaldehyde emission from comet Halley', Astron. J., 97, 246-253.

Weaver, H.A., and Mumma, M.J. (1984) 'Infrared molecular emissions from comets', Ap. J., 276, 782-797.

Erratum: (1984) Ap. J., 285, 872-873.

Weaver, H.A., Mumma, M.J., Larson, H.P., and Davis, D.S. (1986) 'Post-Perihelion observations of water in comet Halley', Nature, 324, 441-444.

Weaver, H.A., Mumma, H.A., and Larson, H.P. (1987) 'Infrared investigation of water in comet P/Halley', Astron. Ap., 187, 411-418.

Wyckoff, S., Tegler, S., and Engel, L. (1990) 'The ammonia abundance in four comets', Ap. J., submitted.

Yamamoto, T. (1982) 'Evaluation of infrared line emission from constituent molecules of cometary nuclei', Astron. Ap., 109, 326-330. 